\title{
Evaluation of land suitability for citrus cultivation in Khana Local Government Area of Rivers State, Southern Nigeria
}

\author{
Kaananwii Dum Peter ${ }^{1^{*}}$ and Aigboghosa Samson Umweni ${ }^{2}$ \\ ${ }^{1}$ Department of Crop/Soil Science, Faculty of Agriculture, Rivers State University \\ P.M.B 5080, Port Harcourt, Rivers State, Nigeria \\ ${ }^{2}$ Department of Soil Science and Land Resource Management, Faculty of Agriculture, University of Benin \\ P.M.B 1154, Ugbowo Lagos Rd, Benin City, Edo State, Nigeria \\ *Corresponding author: dumkapbase@yahoo.com
}

\section{Article Info}

Received : 02 ${ }^{\text {nd }}$ October 2020 Revised : $23^{\text {rd }}$ November 2020

Accepted: $01^{\text {st }}$ December 2020

\section{Keywords:}

Mapping units, marginally suitable, nonparametric method, not suitable, semi detailed soil survey

\begin{abstract}
Soils of Khana Local Government Area of Rivers State, Southern Nigeria were evaluated using semi detailed soil survey for citrus cultivation. The purpose of this research was to evaluate the suitability of soils of the study area for the cultivation of citrus. The total land area covers 49,631.54 ha and was delineated into eight mapping units based on the soil types. One pedon each was dug in each mapping unit and described using the FAO system. The nonparametric method of soil suitability evaluation was used. Four soil orders, majorly Inceptisols/Cambisols, Entosols/Arenosols, Ultisols/Acrisols, and Alfisols/Lixisols, were identified in the area. The results showed that land requirements/characteristics such as climate (mean annual rainfall), wetness (depth to water table) and fertility made the land marginally suitable (S3) to not suitable (N) for citrus cultivation across the eight pedons. The three limitations for citrus cultivation in the area are climate (annual rainfall), wetness (depth to the water table) and fertility (low status of NPK and pH). The land is potentially suitable for citrus cultivation but currently marginally and not suitable due to these three limitations.
\end{abstract}

\section{INTRODUCTION}

Land suitability evaluation is best described as the process of estimating the agricultural land potential for diverse kinds of utilization on a sustainable basis (Peter and Umweni, 2020a). According to Bintang and Tampubolon (2018), land suitability assessment is done by matching land qualities and characteristics to the criteria of the land suitability classes. Land suitability evaluation is also the assessment of agricultural land resources aiming to optimize land productive potentials, adding that land evaluation provides key information on the ability of land for sustainable crop production and soil management (Chukwu et al., 2014; Peter and Umweni, 2020a). It is also the interpretation of soil survey data in order that every hectare of land could be used in accordance with its capability, suitability and limitations (Food and Agriculture Organization, 2006). Soil suitability assessment involves a scientific procedure, which is essential to assess the potential and constraints of a given land for agricultural purposes (Rossiter, 1996). Therefore, to maintain sustainable agriculture, land use planning should be undertaken by investigating the soil through land suitability evaluation studies at both local and regional levels (Sereke, 2002; Essoka and Essoka 2013; Douglas and Peter, 2016; and Peter and Umweni, 2020a). Again, knowledge of the potentials and limitations of agricultural land resources in Khana Local Government Area, will enable crop farmers in the area to make adequate land use initiative to improve and maintain high yield of citrus crops on a sustainable basis and, at the same time, improve their standard of living (Peter and Umweni,

How to cite: Peter, K.D. and Umweni, A.S. (2021). Evaluation of land suitability for citrus cultivation in Khana Local Government Area of Rivers State, Southern Nigeria. IImu Pertanian (Agricultural Science), 6(1), pp. 1-9 
2020b). This requires a proper organization of land and soil data in such a way that they could be interpreted and applied for sustainable agricultural production. Citrus are predominantly produced on large scale within the Middle belt in Nigeria and have enormous market value with very high sales, especially within the dry season. Citrus is an important crop due to its nutritional and medicinal values. There are different citrus cultivars cultivated in Nigeria, such as sweet orange (Citrus sinesis Osbeck), lime (Citrus aurantifolia Swingle), tangerine or tangor (Citrus nobilis) and (Citrus reticulate, Blanco). Therefore, this study aimed to evaluate the suitability of agricultural land resources for citrus cultivation in Khana Local Government Area of Rivers State, Southern Nigeria on a sustainable basis.

\section{MATERIALS AND METHODS}

The study was carried out in Khana Local Government Area of Rivers State, Southern Nigeria within the Tropical Rainforest zone. It is located between latitude 4.67172N and longitude 7.34398E (Figure 1) (Peter and Umweni, 2020b). The study location covers $49,631.54$ ha of land with a rainfall pattern that is in a bimodal form that usually start effectively from late February to October with a period of low precipitation in August commonly called August break (Peter and Ayolagha, 2012). The period of effective low precipitation occured mainly from late November to early March. Sometimes, it is accompanied by serious dry cold wind commonly called harmathan wind. The average rainfall of the study location was between $2000 \mathrm{~mm}$ to $2500 \mathrm{~mm}$ with monthly temperature range of $26^{\circ} \mathrm{C}$ to $35^{\circ} \mathrm{C}$ and relative humidity varying from $81 \%$ to $87 \%$ depending on the season (rainy season and dry season) (Peter and Umweni, 2020b).

\section{Field work}

The entire land of Khana LGA was identified and delineated into eight mapping units based on vegetation, topography, soil types, drainage condition, textures and structures. One soil profile pit of $2 \mathrm{~m} \times 2 \mathrm{~m} \times 2 \mathrm{~m}$ was dug in each representative soil mapping unit. Each of the soil profile was described in line with soil procedure as recommended by Food and Agriculture Organization (1988). Soil samples were collected from identifiable horizons in each of the profile pit for physical and chemical analysis. Undisturbed core samples were collected from each identified profile pit horizon for bulk density determinations. The coordinates of all profile pits were collected using a hand-held geographical positioning system (GPS). Soil color notation in the field was described using the Munsell color chart (1992).

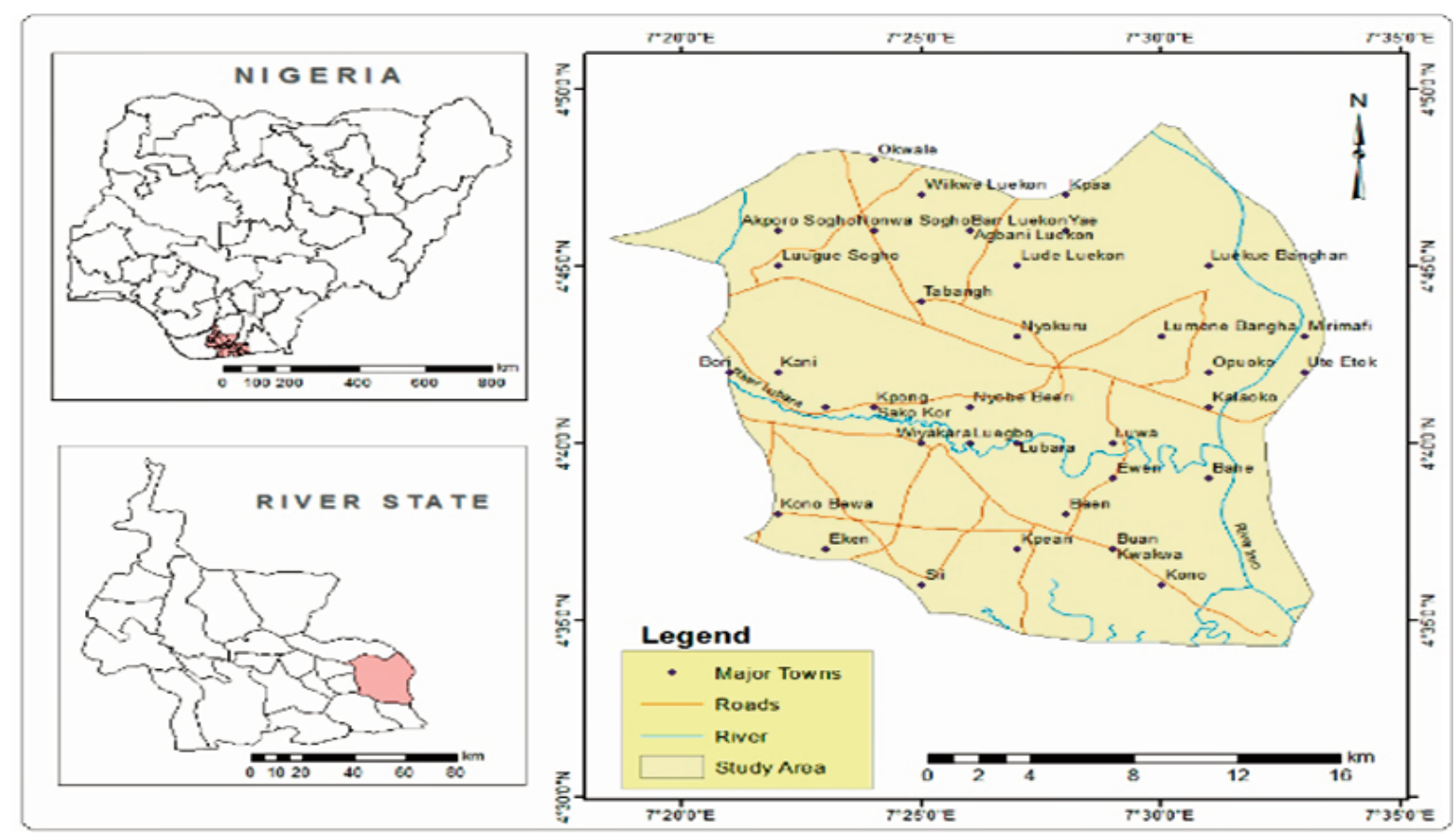

Figure 1. Map of Khana Local Government Area (Project Site). Sources: Government of Rivers State, Office of Surveyor General (2014) 


\section{Laboratory analysis}

Soil samples collected were analyzed using routine soil analysis procedures most appropriate at the Soil Science Laboratory, Federal University Technology, Owerri - Imo State, to determine the physical and chemical characteristics of the soils. Soils collected were air-dried, crushed gently and sieved with a 2 $\mathrm{mm}$ mesh sieve. Soil particle size distributions were determined using the hydrometer method of Bouyoucus (1962) and Blake (1965), bulk density was determined by oven drying the undisturbed soil samples collected using a cylindrical core samplers, and bulk density was calculated as the weight of the soils divided by the volume of soil sample. Soil reaction $(\mathrm{pH})$ was determined using the glass electrode $\mathrm{pH}$ meter (Mclean 1965). Organic carbon was determined using the dichromate wet oxidation method (Walkey and Black, 1934). Organic matter was obtained by multiplying the percentage of organic matter by 1.72 . Total nitrogen was determined by the macro-Kjeldahl digestion methods by Jackson (1973) as described by Bremner and Mulvaney (1982). Available phosphorus was determined by Bray and Kurtz No 2 method (1945). Exchangeable cations were determined by extracting them with neutral ammonium acetate

Table 1. Soil-site suitability criteria for citrus production

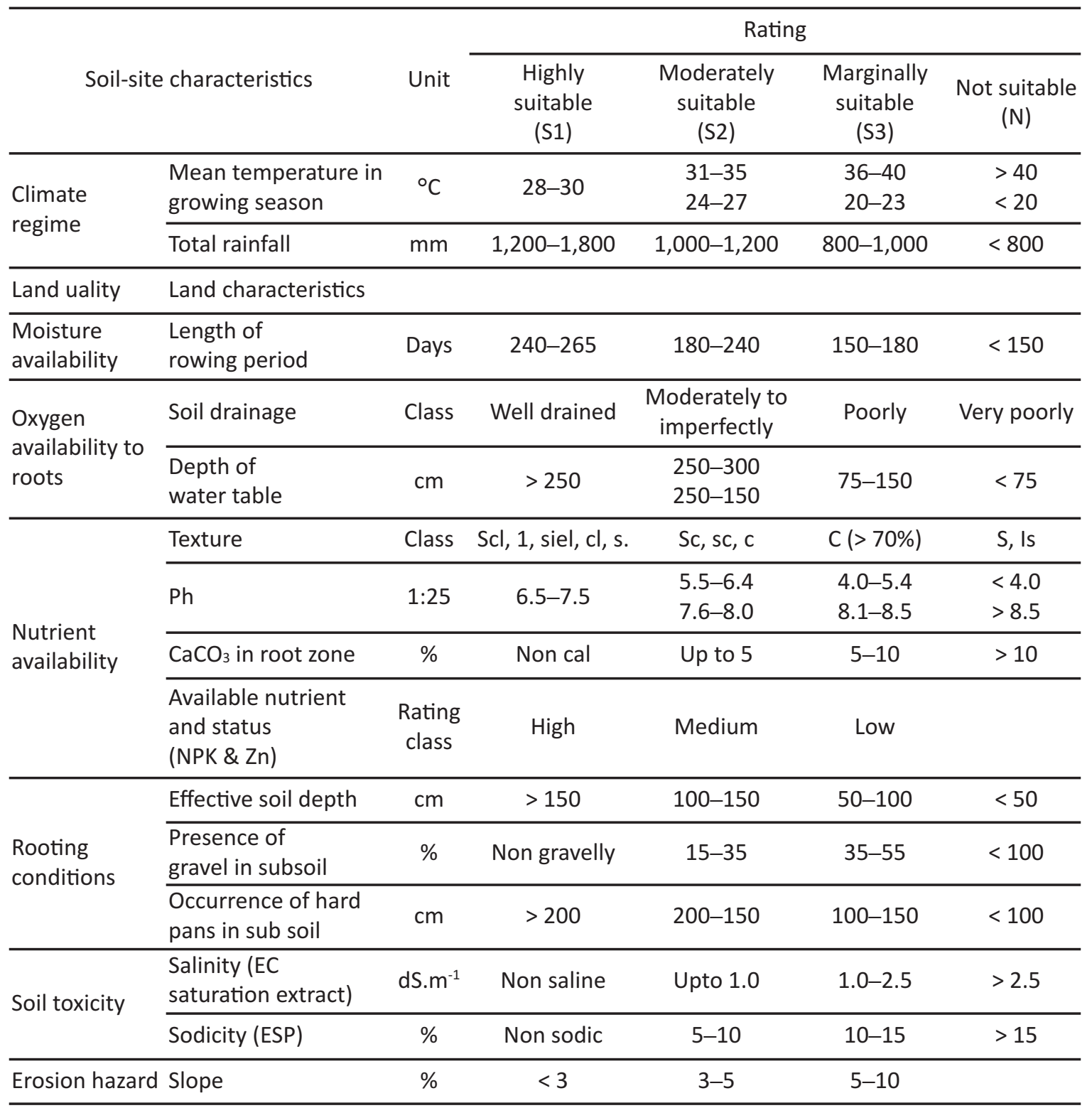

Remark: Irrigation sources are mandatory for successful crop; Source: Mohekar (1997). 
(IM NH${ }_{4} \mathrm{OAc}$ ) buffered at $\mathrm{pH}$ 7.0. Exchangeable cations ( $\mathrm{Ca}, \mathrm{Mg}, \mathrm{K}$ and $\mathrm{Na}$ ) were leached from the soil with $\mathrm{NH}_{4} \mathrm{OAc}$ solution. $\mathrm{Na}$ and $\mathrm{K}$ were determined with flame photometry; while Ca and Mg were determined by the EDTA titration method of Heald (1965). Base saturation was calculated as the sum of exchangeable bases divided by ECEC multiplied by 100 .

\section{Soil classification}

Based on the results of the laboratory analysis and field morphological characteristics, the eight pedons were classified according to soil taxonomy United States Department of Agriculture (2014) and correlated with the World Reference Base for Soil Resources (2014).

\section{Land suitability evaluation procedure}

Soil characteristics of each soil mapping unit were matched with the requirement of the land qualities (climate, topography, wetness, soil physical characteristics, and fertility) to a suitability class assigned to it, following the guidelines provided by Mohekar (1997), (Table 1 and Table 2). The final suitability class of each mapping unit is the class indicated by the characteristics with the lowest ranking, which is in line with the "Law of Minimum" (Food and Agriculture Organization, 1984). The suitability classes of each mapping unit were ranked, and the rankings were compared using Spearman's ranking correlation coefficient.

\section{RESULTS AND DISCUSSION}

\section{Physical and chemical characteristics of the soils}

Table 3 showed the selected physical and chemical characteristics of soils in the study area. Sand size particle dominated other soil particles in all the eight Pedons. Sand particles varied from (705.5 to 792.4) g.kg-1 in Pedon 1, (833.6 to 863.6) g. kg-1 in Pedon 2, (712.6 to 802.4) g. $\mathrm{kg}^{-1}$ in Pedon 3, (792.4 to 802.4) g. kg ${ }^{-1}$ in Pedon 4, (702.2 to 802) g. $\mathrm{kg}^{-1}$ in pedon 5, (760 to 781.6) g.kg-1 in Pedon 6, (691.2 to 812.4) g.kg-1 in Pedon 7 and (813.6 to 853.6) g. $\mathrm{kg}^{-1}$ in Pedon 8, accordingly. The high-level sand in surface horizons is also in line with the report of Akamigbo and Asadu (1983), who reported that sand particles were observed more in surface level as a result of the

Table 2. Factor rating of land quality for the Thanh Tra" Pomelo (Citrus)

\begin{tabular}{|c|c|c|c|c|c|c|}
\hline \multirow{2}{*}{ Land quality } & \multirow{2}{*}{ Diagnostic factor } & \multirow{2}{*}{ Unit } & \multicolumn{4}{|c|}{ Factor rating } \\
\hline & & & S1 & S2 & S3 & $\mathrm{N}$ \\
\hline \multirow{2}{*}{ Temperature } & \multirow{2}{*}{$\begin{array}{l}\text { Mean temperature } \\
\text { in growing season }\end{array}$} & \multirow{2}{*}{${ }^{\circ} \mathrm{C}$} & \multirow{2}{*}{$5-30$} & $30-33$ & $30-35$ & $>15$ \\
\hline & & & & $25-18$ & $18-13$ & $<13$ \\
\hline \multirow{2}{*}{$\begin{array}{l}\text { Water } \\
\text { availability }\end{array}$} & \multirow{2}{*}{$\begin{array}{l}\text { Average annual } \\
\text { rainfall }(R)\end{array}$} & \multirow{2}{*}{$\mathrm{mm}$} & \multirow{2}{*}{$1,500-2,000$} & \multirow{2}{*}{$2,000-2,500$} & $2,500-3,000$ & $>1,000$ \\
\hline & & & & & $1,100-1,200$ & $<1,100$ \\
\hline \multirow{6}{*}{$\begin{array}{l}\text { Fertility } \\
\text { availability }\end{array}$} & Organic carbon (C) & $\%$ & $>25$ & $1.0-2.5$ & $<1.0$ & - \\
\hline & Total nitrogen $(\mathrm{N})$ ) & $\%$ & $>0.2$ & $0.1-0.2$ & $<0.1$ & - \\
\hline & $\begin{array}{l}\text { Available } \\
\text { phosphorus }\left(\mathrm{P}_{2} \mathrm{O}\right)\end{array}$ & ppm & $>15$ & $6-15$ & $<6$ & - \\
\hline & $\begin{array}{l}\text { Available potassium } \\
\left(\mathrm{K}_{2} \mathrm{O}\right)\end{array}$ & mg & $>15$ & $10-15$ & $<10$ & - \\
\hline & \multirow{2}{*}{ Soil reaction $(\mathrm{pH})$} & \multirow{2}{*}{-} & \multirow{2}{*}{$5.5-6.5$} & $6.5-7.5$ & $7.5-8.5$ & $>8.4$ \\
\hline & & & & $5.0-5.5$ & $4.0-5.0$ & $<4.0$ \\
\hline $\begin{array}{l}\text { Rooting } \\
\text { condition } \\
\end{array}$ & Soil depth (D) & $\mathrm{cm}$ & $>100$ & $70-100$ & $50-70$ & $<50$ \\
\hline Erosion hazard & Slope gradient (SL) & degree & $0-30$ & $3-80$ & $8-150$ & $>150$ \\
\hline $\begin{array}{l}\text { Water and } \\
\text { nutrition } \\
\text { retention }\end{array}$ & Soil texture $(T)$ & - & L, LS & SL, SiL & $\mathrm{Si}, \mathrm{CL}$ & $\mathrm{S}, \mathrm{C}$ \\
\hline
\end{tabular}

Remarks: L= Loam, LS= Loam sand; Si= Silt. SiL= Silt loam; S= Sand, SL = Sand loam, C = Clay, CL = Clay loam SI = Highly suitable, $\mathrm{S} 2=$ Moderately suitable, $\mathrm{S} 3=$ Marginally suitable, $\mathrm{N}=$ Not suitable 
Table 3. Morphological and physical characteristics of soils of the study area

\begin{tabular}{|c|c|c|c|c|c|c|c|c|c|c|c|c|c|}
\hline $\begin{array}{l}\text { Pedon } \\
\text { design }\end{array}$ & $\begin{array}{l}\text { Horizon } \\
\text { depth }\end{array}$ & Color (moist) & TC & Structure & Consistence & Drainage & Boundary & Roots & Sand & $\begin{array}{l}\text { Silt } \\
\text { g.kg-1 }\end{array}$ & $\stackrel{\text { Clay }}{\longrightarrow}$ & $\begin{array}{l}\mathrm{BD} \\
\mathrm{g} \cdot \mathrm{cm}^{-3}\end{array}$ & $\begin{array}{l}\text { TP } \\
\%\end{array}$ \\
\hline \multicolumn{14}{|c|}{ PEDON 1} \\
\hline A & $0-18$ & 7.5 YR $3 / 3$ (DB) & $S L$ & G & Friable & WD & CS & $\mathrm{M} 2 \mathrm{rts}$ & 792.4 & 40 & 167.4 & 1.4842 & 44.2 \\
\hline AB1 & $18-40$ & $10 Y 4 / 3(D G B)$ & SL & G & Friable & WD & Diffused & $\mathrm{Cl} \mathrm{rts}$ & 772.4 & 50 & 177.6 & 1.623 & 38.98 \\
\hline AB2 & $40-73$ & 10 YR $5 / 2(G B)$ & $S L$ & G & Friable & WD & Diffused & Fl rts & 762.6 & 70 & 167.4 & 1.841 & 30.79 \\
\hline BW1 & $73-102$ & 10 YR 4/4 (DYB) & SL & Crumb & Loose & WD & Diffused & VFI rts & 706.5 & 120 & 174.5 & 1.334 & 49.84 \\
\hline BW2 & $102-131$ & 10 YR 5/4 (YB) & LS & $\mathrm{SAB}$ & Firm & PD & CS & VF1Yts & 772.6 & 90 & 137.4 & 1.7492 & 32.72 \\
\hline \multicolumn{14}{|c|}{ PEDON 2} \\
\hline A & $0-12$ & 10 YR 4/1 (DG) & LS & $\mathrm{FG}$ & Friable & WD & AW & $\mathrm{M} 2 \mathrm{rts}$ & 833.6 & 103.6 & 62.8 & 1.478 & 44.44 \\
\hline AB1 & $12-26$ & 7.5 YR3/2(VDG) & LS & G & Friable & WD & CS & F2 rts & 863.6 & 53.6 & 82.8 & 1.346 & 49.4 \\
\hline AB2 & $26-40$ & 2.5 YR $5 / 2(G)$ & LS & Crumby & Loose & WD & CS & Vfl rt's & 833.6 & 73.6 & 92.8 & 1.566 & 41.33 \\
\hline BW1 & $40-99$ & $10 \mathrm{YR}^{3 / 4}(\mathrm{LB})$ & LS & $\mathrm{SAB}$ & Loose & WD & AW & Vfl rts & 833.6 & 43.6 & 122.8 & 1.512 & 43.16 \\
\hline BW2 & $99-200$ & $10 \mathrm{YR}^{3} 3 / 4(\mathrm{YB})$ & LS & SAB & Loose & WD & AW & vf2 $\mathrm{rts}$ & 843.6 & 23.6 & 132.8 & 1.731 & 34.94 \\
\hline \multicolumn{14}{|c|}{ PEDON 3} \\
\hline A & $0-23$ & 7.5 YR 4/1 (DB) & LS & SBK & Firm & WD & CS & $\mathrm{M} 2 \mathrm{rts}$ & 802.4 & 50 & 147.6 & 1.5080 & 42.8 \\
\hline$A B$ & $23-60$ & 7.5 YR $4 / 4(B)$ & SL & SBK & Firm & WD & CS & $\mathrm{f} 2 \mathrm{rts}$ & 782.5 & 60 & 157.5 & 1.7320 & 36.2 \\
\hline B & $60-78$ & 7.5 YR 5/8 (DYB) & SL & SBK & Firm & WD & CS & $\mathrm{f} 2 \mathrm{rts}$ & 762.4 & 50 & 187.6 & 1.8701 & 34.26 \\
\hline $\mathrm{Bt}$ & $78-145$ & 10 YR $6 / 8$ (PY) & $\mathrm{SCL}$ & SBK & Firm & WD & CS & vf1 rts & 722.4 & 70 & 207.6 & 1.7492 & 37.32 \\
\hline Bt2 & $145-200$ & $10 \mathrm{YR} 7 / 8$ (YB) & $\mathrm{SCL}$ & SBK & Firm & WD & CS & vf1 rts & 712.6 & 60 & 227.4 & 2.8920 & 30.20 \\
\hline \multicolumn{14}{|c|}{ PEDON 4} \\
\hline$A$ & $0-23$ & 7.5 YR 5/3(DB) & LS & FG & Loose & $\mathrm{PD}$ & CS & M1rtC & 802.4 & 60.2 & 137.4 & 1.4892 & 46.24 \\
\hline Abw & $23-50$ & 7.5 YR $4 / 3$ (B) & LS & FG & Loose & PD & CS & M1rts & 792.4 & 60.2 & 147.4 & 2.3050 & 31.80 \\
\hline \multicolumn{14}{|c|}{ PEDON 5} \\
\hline$A$ & $0-13$ & 7.5 YR $3 / 2$ (DB) & LS & Crumby & Firm & WD & CS & M2rts & 802 & 140.1 & 57.9 & 1.6501 & 40.32 \\
\hline$A B$ & $13-46$ & 7.5 YR $3 / 4$ (DB) & SL & SBK & Firm & WD & CS & $\mathrm{f} 2 \mathrm{rts}$ & 762.2 & 150 & 87.8 & 1.7440 & 36.30 \\
\hline AW1 & $46-71$ & 7.5 YR 4/6 (SB) & SL & SBK & Firm & PD & CS & vf1 rts & 722.4 & 120.2 & 157.4 & 1.7459 & 37.24 \\
\hline BW2 & $71-120$ & 7.5 YR $6 / 4$ (LB) & SL & SBK & Firm & $\mathrm{PD}$ & CS & vf1 rts & 702.2 & 100.2 & 197.6 & 1.8016 & 34.62 \\
\hline \multicolumn{14}{|c|}{ PEDON 6} \\
\hline A & $0-26$ & 7.5 YR 4/1 (DG) & LS & SBK & Friable & WD & CS & $\mathrm{M} 2 \mathrm{rts}$ & 781.6 & 154 & 64.4 & 1.4401 & 50.20 \\
\hline$A B$ & $26-52$ & 7.5 YR $3 / 1$ (VDG) & LS & SBK & Friable & WD & CS & $\mathrm{C} 12 \mathrm{rts}$ & 760 & 140 & 100 & 1.4926 & 47.28 \\
\hline B & $52-114$ & 7.5 YR $4 / 4$ (DYB) & $\mathrm{SCL}$ & SBK & Firm & WD & CS & $\mathrm{C} 2 \mathrm{rts}$ & 720.4 & 140 & 139.6 & 1.6012 & 41.16 \\
\hline $\mathrm{Bt}$ & $114-200$ & 7.5 YR 6/4 (LYB) & $\mathrm{SCL}$ & SBK & Firm & WD & CS & vf2 $\mathrm{rts}$ & 702.4 & 91.2 & 206.4 & 1.7309 & 36.1 \\
\hline \multicolumn{14}{|c|}{ PEDON 7} \\
\hline A & $0-17$ & 10 YR $4 / 1$ (DG) & SL & Crumby & Loose & WD & CS & $\mathrm{M} 2 \mathrm{rts}$ & 812.4 & 110 & 77.6 & 1.6081 & 24.44 \\
\hline$A B$ & $17-35$ & 10 YR 5/4 (DYB) & SL & SBK & Firm & WD & CS & $\mathrm{f} 2 \mathrm{rts}$ & 801.6 & 101.4 & 97 & 1.8624 & 35.27 \\
\hline B & $35-78$ & 10 YR 4/6 (PYB) & SL & SBK & Firm & WD & CS & vf1 rts & 760 & 140 & 100 & 2.3152 & 30.64 \\
\hline $\mathrm{Bt}$ & $78-200$ & 10 YR 6/4 (LYB) & $\mathrm{SCL}$ & SBK & Firm & WD & CS & vf1 rts & 691.2 & 60.2 & 248.6 & 1.5022 & 43.76 \\
\hline \multicolumn{14}{|c|}{ PEDON 8} \\
\hline$A$ & $0-22$ & 10 YR $2 / 2$ (VDB) & LS & Granular & Friable & WD & CS & $\mathrm{M} 2 \mathrm{rts}$ & 853.6 & 53.6 & 92.8 & 1.537 & 42.22 \\
\hline Ah & $22-36$ & 10 YR $4 / 3$ (B) & LS & Granular & Friable & WD & CS & $1 \mathrm{rts} C$ & 823.6 & 53.6 & 112.8 & 1.568 & 41.05 \\
\hline$A B$ & $36-50$ & 10 YR $6 / 3$ (LYB) & SL & Crumby & Loose & WD & CS & vf 2 rts & 823.6 & 43.6 & 132.8 & 1.581 & 40.56 \\
\hline B & 50-109 & 10 YR $5 / 3$ (LB) & SL & SBK & Firm & WD & CS & vf1 rts & 813.6 & 63.6 & 122.8 & 1.663 & 37.48 \\
\hline BW & $109-200$ & 10 YR 5/6 (PYB) & SL & SBK & Firm & WD & CS & vf1 rts & 813.6 & 63.6 & 122.8 & 1.683 & 36.71 \\
\hline
\end{tabular}

Remarks: $\mathrm{DB}=$ Dark brown, $\mathrm{DGB}=$ Dark gray brown, $\mathrm{GB}=$ Gray brown, $\mathrm{DYB}=$ Dark yellowish brown, $\mathrm{YB}=$ Yellowish brown, $\mathrm{SL}=\mathrm{Sandy}$ loam, $\mathrm{LS}=$ Loamy sand, $\mathrm{G}=\mathrm{Granular}, \mathrm{SAB}$ Sub-angular blocky, $\mathrm{WD}=$ Well drained, $\mathrm{PD}=$ Poorly drained, $\mathrm{CS}=\mathrm{Clear}$ smooth, $\mathrm{M}=$ Many, $\mathrm{I}=$ Fine, $2=$ Medium, $\mathrm{F}=$ Few, $\mathrm{C}=$ Common, $\mathrm{VF}=$ Very few, $\mathrm{rts}=$ roots. $\mathrm{TC}=$ Textural class, $\mathrm{BD}=\mathrm{Bulk}$ density and $\mathrm{TP}=$ Total porosity.

eluviation and illuviation processes in soils. The high sand fraction in surface horizon was also influenced by the parent material from which the soils are formed (Akpan-Idiok (2012); Peter and Umweni, 2020b). There were some degrees of variability in silt contents in all the eight pedons. It varied from (40 to 120 ) g. $\mathrm{kg}^{-1}$ in Pedon 1, (23.6 to 103.6) g. $\mathrm{kg}^{-1}$ in Pedon 2, (50 to 70) g. $\mathrm{kg}^{-1}$ in Pedon 3, $60.2 \mathrm{~g}^{\mathrm{kg}} \mathrm{kg}^{-1}$ in Pedon 4, (100.2-140.1) g. $\mathrm{kg}^{-1}$ in Pedon 5, (702.4 to 781.6) g. kg ${ }^{-1}$ in Pedon 6, (60.2-10) g.kg-1 in Pedon 7 and (53.6 to 63.6) g. kg ${ }^{-1}$ in Pedon 8. Clay content of soils in the study area also varied between (137.4 to 177.6) g. $\mathrm{kg}^{-1}$ in Pedon 1, (62.8 to 132.8) g. $\mathrm{kg}^{-1}$ in Pedon 2, (147.6 to 227.4) g. kg-1 in Pedon 3, (137.4 to 147.4) g. $\mathrm{kg}^{-1}$ in Pedon 4, (57.9 to 197.6) g. $\mathrm{kg}^{-1}$ in Pedon 5,

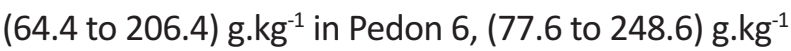

in Pedon 7 and (92.8 to 132.8 ) g. $\mathrm{kg}^{-1}$ in Pedon 8 accordingly (Peter and Umweni, 2020b). Soil reactions $(\mathrm{pH})$ in water, as shown in Table 4, varied from acidic (4.31) to slightly acidic (6.16). Soil pH increased from 5.43 to 6.13 in pedon 1 and increased from 5.6 to 6.16 in pedon 2. It also increased from 5.4 to 6.11 in pedon 3. It was also observed that soil pH increased from 5.43 to 6.08 in pedon 4 and increased from 4.50 to 5.71 in pedon 5 . In pedon 6 , there was an increase from 4.31 to 4.81 , and in pedon 7 , there was a decrease from 5.9 to 4.7 and an increase from 5.59 to 5.83 in pedon 8. There was a decrease in soil $\mathrm{pH}$ down the profile depth, which is in line with the finding of Peter and Umweni (2020b). Soil organic carbon was generally low in all pedons (1.40 to 14.15 $\left.\mathrm{g} \cdot \mathrm{kg}^{-1}\right)$. This is also in line with the findings of Thurow 
Table 4. Chemical characteristics of soils of the study area

\begin{tabular}{|c|c|c|c|c|c|c|c|c|c|c|c|c|c|c|c|c|c|}
\hline & $\begin{array}{l}\text { Depth } \\
(\mathrm{cm})\end{array}$ & $\left(\mathrm{H}_{2} \mathrm{O}\right)$ & $\longleftarrow$ & $\begin{array}{c}\text { OM } \\
\text { g.kg-1 }\end{array}$ & $\mathrm{TN}$ & $\begin{array}{l}\text { Alv. P } \\
\mathrm{mg} \cdot \mathrm{kg}^{-1}\end{array}$ & $\mathrm{Ca}^{2+}$ & $\mathrm{Mg}^{2+}$ & $\mathrm{kg}^{-1}$ & $\mathrm{Na}^{+}$ & Ca:Mg & $\mathrm{EA} 1^{3+}$ & $\mathrm{EH}^{+}$ & TEA & ECEC & $\mathrm{C}: \mathrm{N}$ & BS \\
\hline \multicolumn{18}{|c|}{ PEDON 1 Oxyaquic Dystrudept/ Stagnic Endogleyic Cambisol (Oxyaquic, Hyperdystric) } \\
\hline A & $0-18$ & 5.66 & 14.15 & 24.59 & 1.39 & 10.88 & 0.78 & 0.40 & 0.200 & 0.183 & $2: 1$ & 1.76 & 1.04 & 2.80 & 4.483 & 10:01 & 34.865 \\
\hline$A B_{1}$ & $18-40$ & 5.43 & 11.11 & 19.15 & 1.08 & 15.63 & 1.0 & 0.81 & 0.189 & 0.162 & $1: 1$ & 1.84 & 1.65 & 3.49 & 5.651 & 10:01 & 38.039 \\
\hline $\mathrm{AB}_{2}$ & $40-73$ & 6.08 & 12.08 & 20.83 & 1.04 & 9.27 & 1.0 & 0.82 & 0.177 & 0.135 & $1: 1$ & 1.15 & 1.03 & 2.18 & 4.312 & 12:01 & 49.44 \\
\hline $\mathrm{BW}_{1}$ & $73-102$ & 6.13 & 5.13 & 9.75 & 0.54 & 12.43 & 0.60 & 0.41 & 0.170 & 0.140 & $2: 1$ & 0.84 & 0.52 & 1.36 & 2.68 & 10.43:1 & 49.254 \\
\hline \multicolumn{18}{|c|}{ PEDON 2 Typic Udipsamment/Haplic, Hypoferralic Arenosol (Hyperdystric) } \\
\hline A & $0-12$ & 6.16 & 10.57 & 18.23 & 1.09 & 6.23 & 0.63 & 0.23 & 0.301 & 0.607 & $2: 1$ & 1.14 & 0.496 & 1.64 & 3.408 & $9.70: 1$ & 51.86 \\
\hline$A B$ & $12-26$ & 5.94 & 8.28 & 14.27 & 0.89 & 4.76 & 0.37 & 0.12 & 0.254 & 0.47 & $3: 1$ & 0.624 & 0.432 & 1.056 & 2.280 & $9.30: 1$ & 54.1 \\
\hline B & $26-40$ & 5.96 & 4.39 & 7.57 & 0.39 & 2.94 & 0.19 & 0.05 & 0.277 & 0.411 & $4: 1$ & 0.720 & 0.432 & 1.152 & 2.06 & 19.4:1 & 46.65 \\
\hline $\mathrm{BW}_{1}$ & $40-99$ & 6.05 & 3.09 & 5.33 & 0.36 & 9.17 & 0.10 & 0.02 & 0.276 & 0.366 & $5: 1$ & 0.768 & 0.480 & 1.248 & 2.01 & $8.58: 1$ & 37.91 \\
\hline \multicolumn{18}{|c|}{ PEDON 3 Oxyaquic Dystrudept/Plinthic Endogleyic Cambisol (Oxyaquic, dystric) } \\
\hline A & $0-23$ & 6.08 & 10.06 & 17.34 & 0.90 & 39.08 & 1.50 & 0.95 & 0.198 & 0.220 & $1.58: 1$ & 1.76 & 1.48 & 3.24 & 6.108 & 10.59:1 & 46.955 \\
\hline$A B$ & $23-60$ & 5.14 & 8.15 & 14.05 & 0.63 & 26.19 & 0.10 & 0.40 & 0.180 & 0.202 & $2.25: 1$ & 1.44 & 1.40 & 2.84 & 4.522 & 12.93:1 & 34.104 \\
\hline B & $60-78$ & 6.11 & 6.26 & 10.79 & 0.59 & 14.23 & 1.00 & 0.36 & 0.161 & 0.190 & $2.78: 1$ & 1.32 & 1.05 & 2.37 & 4.081 & 10.61:1 & 41.926 \\
\hline $\mathrm{Bt}_{2}$ & $78-145$ & 5.28 & 4.18 & 7.21 & 0.42 & 12.64 & 1.10 & 0.56 & 0.142 & 0.183 & $1.96: 1$ & 2.16 & 2.12 & 4.28 & 6.265 & $9.95: 1$ & 47.647 \\
\hline $\mathrm{Bt}_{3}$ & $145-200$ & 5.97 & 2.13 & 3.67 & 0.30 & 13.22 & 0.50 & 0.31 & 0.135 & 0.170 & $1.61: 1$ & 2.11 & 2.03 & 4.14 & 5.255 & 7.1:1 & 21.218 \\
\hline \multicolumn{18}{|c|}{ PEDON 4 Aquic Udipsamment/Haplic Endostagnic Arenosol (Greyic, Hyperdystric) } \\
\hline A & $0-23$ & 5.43 & 10.83 & 18.67 & 1.07 & 20.11 & 1.1 & 0.17 & 0.21 & 0.238 & $1.13: 1$ & 1.01 & 0.95 & 1.96 & 3.678 & 10.12:1 & 34.059 \\
\hline Abw & $23-50$ & 6.08 & 10.17 & 17.53 & 0.98 & 6.44 & 0.80 & 0.72 & 0.116 & 0.128 & 1.11:1 & 1.12 & 1.04 & 2.16 & 3.924 & 10.38 .1 & 44.954 \\
\hline \multicolumn{18}{|c|}{ PEDON 5 Typic Dystrudept/Haplic Ferralic Cambisol (Chromic, Hyperdystric) } \\
\hline A & $0-13$ & 5.71 & 11.1 & 19.14 & 0.41 & 24.19 & 0.10 & 0.38 & 0.181 & 0.186 & $1.58: 1$ & 1.30 & 0.90 & 2.20 & 3.047 & 27.1 & 24.839 \\
\hline$A B$ & $13-46$ & 5.12 & 12.0 & 20.69 & 0.37 & 22.80 & 0.50 & 0.24 & 0.169 & 0.174 & $2.08: 1$ & 1.41 & 2.12 & 3.53 & 4.613 & $327.4: 1$ & 28.750 \\
\hline BW1 & $46-71$ & 4.92 & 8.0 & 13.79 & 0.30 & 21.69 & 1.61 & 0.44 & 0.160 & 0.186 & $3.41: 1$ & 1.31 & 2.06 & 3.57 & 5.766 & 27:1 & 53.228 \\
\hline BW2 & $71-120$ & 4.50 & 6.21 & 10.70 & 0.24 & 9.33 & 1.40 & 0.40 & 0.149 & 0.174 & $3.51: 1$ & 1.9 & 2.12 & 4.02 & 6.484 & 26.1 & 55.228 \\
\hline \multicolumn{18}{|c|}{ PEDON 6 Typic Kandiudults/Haplic Vetic Acrisol (Hyperdystric) } \\
\hline A & $0-26$ & 4.81 & 11.1 & 19.14 & 0.45 & 23.9 & 0.90 & 0.66 & 0.170 & 0.220 & $1.36: 1$ & 1.60 & 1.12 & 2.72 & 3.86 & $2.6: 1$ & 32.397 \\
\hline$A B$ & $26-52$ & 4.62 & 9.11 & 15.71 & 0.31 & 13.14 & 0.60 & 0.48 & 0.170 & 0.208 & $1.25: 1$ & 1.09 & 1.47 & 2.56 & 4.018 & 29.4:1 & 29.889 \\
\hline B & $52-114$ & 4.5 & 3.0 & 5.17 & 0.23 & 14.00 & 0.50 & 0.46 & 0.167 & 0.196 & $1.08: 1$ & 1.10 & 1.08 & 2.18 & 3.503 & 13.4:1 & 32.490 \\
\hline $\mathrm{Bt}$ & $114-200$ & 4.31 & 4.10 & 7.07 & 0.26 & 14.00 & 0.50 & 0.67 & 0.168 & 0.169 & 1.1 & 0.60 & 0.67 & 1.27 & 2.775 & $16: 1$ & 28.809 \\
\hline \multicolumn{18}{|c|}{ PEDON 7 Oxyaquic Kandiudalf/Gleyic Vetic Lixisol (Arenic, Oxyaquic) } \\
\hline A & $0-17$ & 5.9 & 10.01 & 17.26 & 1.12 & 30.88 & 0.90 & 0.66 & 0.196 & 0.194 & $1.36: 1$ & 1.84 & 1.12 & 3.04 & 4.99 & 8.93:1 & 48.653 \\
\hline$A B$ & $17-35$ & 5.7 & 8.06 & 13.99 & 0.86 & 13.09 & 0.92 & 0.44 & 0.161 & 0.182 & $2.09: 1$ & 1.16 & 1.00 & 2.16 & 3.863 & $9.4: 1$ & 30.367 \\
\hline B & $35-78$ & 4.90 & 6.11 & 10.88 & 0.71 & 10.16 & 1.10 & 0.83 & 0.160 & 0.180 & $1.33: 1$ & 0.96 & 1.14 & 2.1 & 4.37 & $8.9: 1$ & 54.035 \\
\hline $\mathrm{Bt}$ & $78-200$ & 4.70 & 4.18 & 7.21 & 0.49 & 9.59 & 1.00 & 0.48 & 0.145 & 0.146 & $2.08: 1$ & 0.84 & 1.03 & 1.89 & 3.641 & $8.53: 1$ & 61.621 \\
\hline \multicolumn{18}{|c|}{ PEDON 8 Fluventic Dystudept/Haplic Fluvic Cambisol (Chromic dystric) } \\
\hline A & $0-22$ & 5.83 & 3.49 & 6.02 & 0.70 & 7.78 & 0.21 & 0.08 & 0.258 & 0.385 & $2.68: 1$ & 0.912 & 0.496 & 1.408 & 2.341 & $5.06: 1$ & 37.85 \\
\hline Ah & $22-36$ & 5.69 & 2.59 & 4.47 & 0.33 & 57.47 & 0.43 & 0.18 & 0.250 & 0.426 & 2.39:1 & 0.592 & 0.368 & 0.96 & 2.246 & $7.85: 1$ & 57.26 \\
\hline$A B$ & $36-50$ & 5.59 & 3.69 & 6.36 & 0.47 & 10.85 & 0.32 & 0.09 & 0.283 & 0.418 & $3.56: 1$ & 0.592 & 0.336 & 0.928 & 2.039 & $4.16: 1$ & 54.49 \\
\hline B & $50-109$ & 5.72 & 3.19 & 5.50 & 0.67 & 1.82 & 0.34 & 0.10 & 0.278 & 0.416 & $3.4: 1$ & 0.448 & 0.176 & 0.624 & 1.758 & 4.76:1 & 54.51 \\
\hline BW & $109-200$ & 5.67 & 1.50 & 2.58 & 0.26 & 51.17 & 0.32 & 0.10 & 0.263 & 0.382 & $2.7: 1$ & 1.088 & 0.328 & 1.416 & 3.439 & $5.77: 1$ & 41.94 \\
\hline
\end{tabular}

and Smith (1998); Essoka and Essoka (2014); and Peter and Umweni, (2020). Total nitrogen levels ranged from very low $\left(0.24 \mathrm{~g}^{\mathrm{kg}}{ }^{-1}\right)$ in pedon 5 to low $\left(1.39 \mathrm{~g} . \mathrm{kg}^{-1}\right)$ in pedon 1 . Total $\mathrm{N}$ decreased down the depth the profile across the pedons. The low level of total $\mathrm{N}$ in the soils in the study area was as a result of excessive soil planted to leaching due to intensive rainfall experienced in the area, supported by the findings of Udo and Ogunwale (1986) \& Peter and Umweni (2020a). Available phosphorus also varied from $1.82 \mathrm{~m} . \mathrm{kg}^{-1}$ (very low) to $57.47 \mathrm{~m} . \mathrm{kg}^{-1}$. There was no decrease in the available $P$ content down the profile depths, but there were differences in the level of available $P$ nonlinear to soil depths in all pedons.

\section{Soil classification}

Four soil orders, majorly Inceptisols/Cambisols, Entosols/Arenosols, Ultisols/Acrisols, and Alfisols/ Lixisols, were identified. According to Peter and Umweni (2020b), they were further classified as
Oxyaquic Dystrudept/Stagnic Endogleyic Cambisol in pedon 1, Typic Udipsamment/Haplic Hypoferralic Arenosol in pedon 2, Oxyaquic Dystrudept/Plinthic Endogleyic Cambisol in pedon 3, Aquic Udipsamment/ Haplic Endostagnic Arenosol in pedon 4, Typic Dystrudept/Haplic Ferralic Cambisol in pedon 5, Typic Kandiudults/Haplic Vetic Acrisol (Hyperdystric) in pedon 6, Oxyaquic Kandiudalf/Gleyic Vetic Lixisol (Arenic, Oxyaquic) in pedon 7, and Fluventic Dystudept/Haplic Fluvic Cambisol (Chromic dystric) in pedon 8 .

\section{Land suitability of the study area for citrus cultivation}

The suitability classification of each pedon in the study area for citrus cultivation showed that Pedons 1 and 5 were currently marginally (S3) suitable for citrus cultivation in the study area due to limitations in climate (rainfall), wetness and fertility (Table 5). This is in line with the findings of Ikhe et al. (2017), who reported that excess rain caused citrus to be waterlogged, which eventually caused molding, 
Table 5. Summary of Suitability Evaluation for Citrus cultivation in Pedons 1-8

\begin{tabular}{|c|c|c|c|c|c|c|c|c|}
\hline \multirow{2}{*}{$\begin{array}{c}\text { Land requirements/Land } \\
\text { suitability } \\
\end{array}$} & \multicolumn{8}{|c|}{ Pedons and their suitability class (s) } \\
\hline & P1 & P2 & P3 & P4 & P5 & P6 & P7 & P8 \\
\hline \multicolumn{9}{|l|}{ Climate } \\
\hline Total rainfall ( $\mathrm{mm}$ & $2,000-2,500(\mathrm{~S} 3)$ & $2,000-25,00(\mathrm{~S} 3)$ & $2,000-2,500(\mathrm{~S} 3)$ & $2,000-2,500(\mathrm{~S} 3)$ & $2,000-2,500(\mathrm{~S} 3)$ & $2,000-2,500(\mathrm{~S} 3)$ & $2,000-2,500(\mathrm{~S} 3)$ & $2,000-2,500(\mathrm{~S} 3)$ \\
\hline Length of growing period (day) & 180 days $(\mathrm{S} 1)$ & 180 days $(\mathrm{S} 1)$ & 180 days $(\mathrm{S} 1)$ & 180 days $(\mathrm{S} 1)$ & 180 days $(\mathrm{S} 1)$ & 180 days $(\mathrm{S} 1)$ & 180 days (S1) & 180 days $(\mathrm{S} 1)$ \\
\hline $\begin{array}{l}\text { Mean temperature in growing } \\
\text { season }\left({ }^{\circ} \mathrm{C}\right)\end{array}$ & $25-28(\mathrm{~S} 1)$ & $25-28(S 1)$ & $25-28(\mathrm{~S} 1)$ & $25-28(S 1)$ & $25-28(S 1)$ & $25-28(\mathrm{~S} 1)$ & $25-28(S 1)$ & $25-28(S 1)$ \\
\hline \multicolumn{9}{|l|}{ Topography } \\
\hline Slope (\%) & $0-4(S 1)$ & $0-4(S 1)$ & $0-4(S 1)$ & $0-4(\mathrm{~S} 1)$ & $0-4(S 1)$ & $0-4(S 1)$ & $0-4(S 1)$ & $0-4(\mathrm{~S} 1)$ \\
\hline Erosion hazard (eh) & Very low (S1) & Very low (S1) & Very low (S1) & Very low (S1) & Very low (S1) & Very low (S1) & Very low (S1) & Very low (S1) \\
\hline \multicolumn{9}{|l|}{ Wetness (W) } \\
\hline Depth of water table $(\mathrm{cm})$ & $131(\mathrm{~s} 3)$ & $>200$ (S1) & $>200$ (S1) & $50(\mathrm{~N})$ & $120(\mathrm{~S} 3)$ & $200(S 1)$ & 200 (S1) & $200(S 1)$ \\
\hline Soil drainage (surface) & $\mathrm{MD}(\mathrm{s} 2)$ & WD (S1) & WD (S1) & $\mathrm{MD}(\mathrm{S} 2)$ & $\mathrm{MD}(\mathrm{s} 2)$ & WD (S1) & WD (S1) & WD (S1) \\
\hline \multicolumn{9}{|l|}{ Soil Physical Characteristic (s) } \\
\hline Texture & LS (S1) & LS (S1) & $\mathrm{SL} \quad(\mathrm{S} 1)$ & SL (S1) & SL (S1) & LS (S1) & SL (S1) & LS (S1) \\
\hline Effective Soil depth & $131(\mathrm{~s} 2)$ & $200(\mathrm{~S} 1)$ & $200(\mathrm{~S} 1)$ & $50(\mathrm{~S} 3)$ & $120(\mathrm{~s} 2)$ & $200(\mathrm{~S} 1)$ & 200 (S1) & 200 (S1) \\
\hline Presence of gravel in subsoil & Nil (S1) & Nil (S1) & Nil (S1) & Nil (S1) & Nil (S1) & Nil (S1) & Nil (S1) & Nil (S1) \\
\hline $\begin{array}{l}\text { Occurrence of Hard Pan in } \\
\text { Subsoil }\end{array}$ & Nil (S1) & NIL (S1) & NIL (S1) & NIL (S1) & NIL (S1) & NIL (S1) & NIL (S1) & NIL (S1) \\
\hline \multicolumn{9}{|l|}{ Fertility } \\
\hline $\mathrm{pH}$ & $5.43-6.13(\mathrm{~s} 2)$ & $5.60-6.16(\mathrm{~s} 2)$ & $5.14-6.11(\mathrm{~s} 2)$ & $5.43-6.06(\mathrm{~S} 2)$ & $4.50-5.71(\mathrm{~S} 3)$ & $4.31-4.81(\mathrm{~S} 3)$ & $4.70-5.70(\mathrm{~s} 3)$ & $5.67-5.83(\mathrm{~S} 2)$ \\
\hline $\begin{array}{l}\text { Availability of nutrient and } \\
\text { status (NPK) }\end{array}$ & Very low (S3) & Very low (S3) & Very low (S3) & Very low (S3) & Very low (S3) & Very low (S3) & Very low (S3) & Very low (S3) \\
\hline Sodicity (Esp) & $0.038(\mathrm{~S} 1)$ & $0.2(\mathrm{~S} 1)$ & $0.036(\mathrm{~S} 1)$ & $0.05(\mathrm{~S} 1)$ & $0.036(\mathrm{~S} 1)$ & $0.06(\mathrm{~S} 1)$ & $0.01(\mathrm{~S} 1)$ & $0.2(\mathrm{~S} 1)$ \\
\hline Aggregate Suitability Class & $(\mathrm{S} 3)(\mathrm{c}, \mathrm{w}, \mathrm{f})$ & $\mathrm{S} 3(\mathrm{c}, \mathrm{f})$ & $\mathrm{S} 3(\mathrm{c}, \mathrm{f})$ & $N(w)$ & S3 $(c, w, f)$ & S3 $(c, f)$ & S3 $(c, f)$ & $S 3(c, f)$ \\
\hline Size (Hectare) & 4,750 & 1,400 & 19,882 & 7,700 & 5,950 & 5,350 & 3,350 & 1,250 \\
\hline$\%$ Coverage & 9.57 & 2.82 & 40.06 & 15.52 & 11.98 & 10.78 & 6.75 & 2.52 \\
\hline
\end{tabular}

Remarks: Pedons 1 and 5 (10,700 ha) were marginally suitable (S3) for citrus cultivation with defects in climate (rainfall), wetness (depth to water table) and fertility (Low N, P and K status) in the soils; Pedons 2, 3, 6, 7, and 8 (31,232 ha) were also marginally suitable (S3) for citrus cultivation but with limitations in both climate and fertility; Pedon $4(7,700)$ was not suitable (N) for citrus cultivation due to limitation in wetness (soil depth to water table); Source : Mohekar (1997).

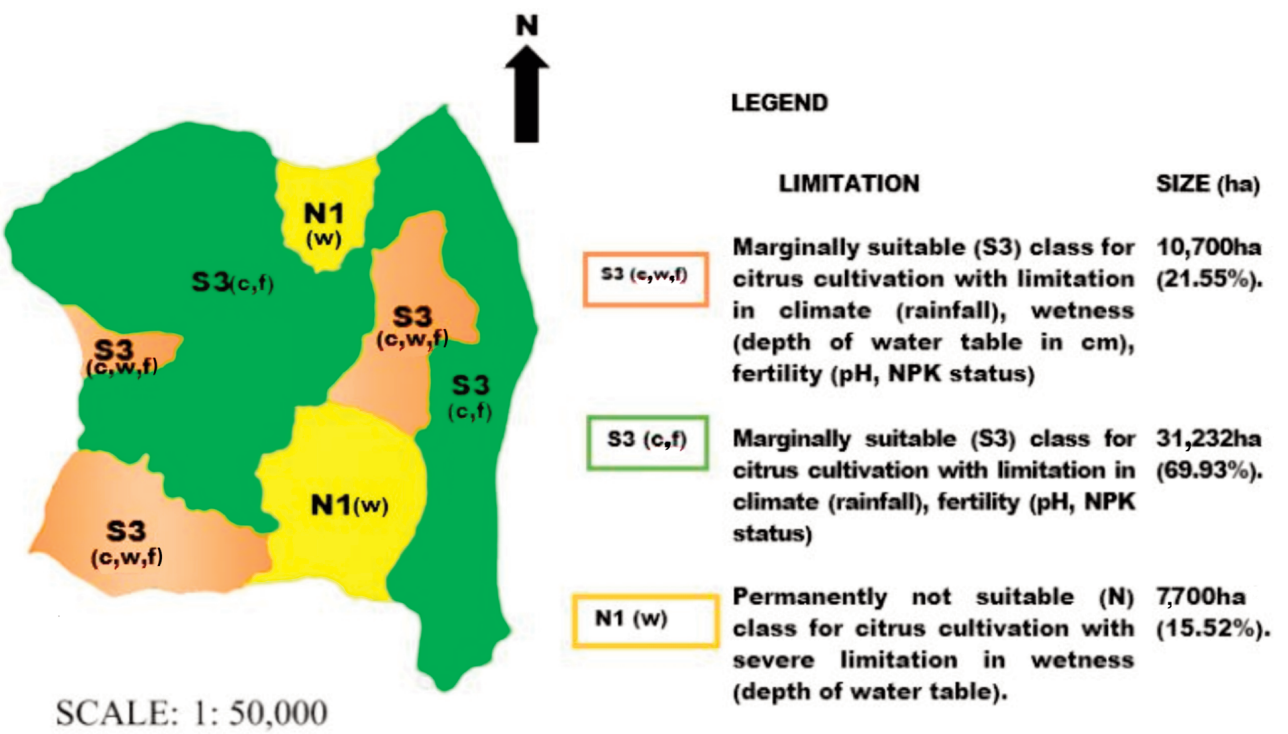

Figure 2. Land Suitability Map for Citrus in Khana Local Government Area.

adding that under such condition, citrus takes in more water and becomes more diluted. Both pedons (1 and 5) cover an area of land of 10,700 ha, representing $21.55 \%$ of the study area. Pedons 2, 3, 6, 7 and 8 were also marginally (S3) suitable with limitations in climate and soil fertility. The main climatic factor affecting citrus production in the area was rainfall. This is synonymous with the finding of Ali et al.
(2017), reporting that rainfall affected the growth and development of citrus in the humid tropical region. They added that excessive rain could lead to the excessive drop of flowers, resulting in fruitless conditions. Pedons $2,3,6,7$, and 8 cover a land area of 31,232 ha, representing $62.93 \%$, of the study area while pedon 4 was permanently not suitable (N) to citrus cultivation due to severity in wetness 
(depth to the water table) (Figure 2). This confirmed the report of Ridolfi (2006), that tree crops were stressed when the depth to the water table was shallow, leading to poor growth and dead of plants due water logging conditions. Pedon 4 covers a land area of 7,700 ha, representing $15.52 \%$ of the study area. The specific fertility limitation was nutrient availability and status, especially NPK, which was very low in all pedons (1-8), while for pedon 4, it was the soil depth to the water table.

\section{CONCLUSIONS}

The land resource (soil) of Khana LGA is marginally suitable (S3), and some were currently not suitable (N) for citrus cultivation due to certain limitations. These limitations ranged from climate (rainfall) to wetness and fertility. A total of $41,931.54$ of land representing $84.49 \%$ of the study area were marginally suitable (S3) for citrus cultivation due to constraints in climate (rainfall), wetness and fertility, while 7,700 ha representing $15.51 \%$ of the study area were permanently not $(\mathrm{N})$ suitable for citrus cultivation due to severity in wetness (depth to the water table). However, some of these limitations can be improved through appropriate management practices in the study area for sustainable citrus cultivation.

\section{REFERENCES}

Akamigbo, F.O.R. and Asadu, C.L.A. (1983). Influence of parent materials on the soils of Southestern Nigeria. East African Agricultural and Forestry Journal, 48(4), pp. 81-91.

Akpan-Idiok, A.U. (2012). Physicochemical properties, degradation rate and vulnerability potentials of soils formed on coastal plain sands in Southeast, Nigeria. International Journal of Agriculture Research, 7(7), pp. 358-366.

Ali, H., Lai, M.I., Nawaz, M.Z., Sharif, M., and Saleem, B.A. (2017). Symptom based automated detection of citrus diseases using color histogram and textural descriptors. Computers and Electronic in Agriculture, 138, pp. 92-104

Bintang, Supriadi, and Tampulobon, E. (2018). Evaluation of land suitability for shallot ( $A$. asculonicum L.) and orange (Orange sp.) at Harian District Samosir Regency. IOP Conference Series: Earth and Environmental Science,
122(1), pp. 012035

Blake, G.R. (1965). Particle density. In: C.A. Blake, ed., Method of Soil Analysis Part 1. Madison, Wisconsin, USA: American Society of Agronomy, pp. 371-373.

Bouyocus, G.H. (1962). Hygrometer method improved for making particle size analysis of soil. Agronomy Journal, 54(5), pp. 464-465.

Bray, R.H. and Kurtz, L.T. (1945). Determination of total and available form of phosphorus in soils. Soil science, 59(1), pp. 39-45.

Bremner, J.M, and Mulvaney, C.S. (1982). Nitrogentotal. In: Page, A.L., Miller, R.H., and Keeney, D.R. eds., Methods of Soil Analysis. Chemical and Microbiological Properties. Madison, Wisconsin, USA : American Society of Agronomy, Soil Science Society of America, pp. 595-624.

Chukwu, G.O., Nwosu, P.O., and Onyekwere, I.N. (2014). Suitability evaluation of land resources zones of Nigeria for cocoyam production. US Open Soil Science Journal, 1(1), pp. 1-8.

Douglas, K. and Peter, K.D. (2016). Assessment of selected soil quality indicators in Obio/Akpor Local Government Area of Rivers State, Southeastern Nigeria. IIARD International Journal of Geography and Environmental Management, 2(8), pp. 10-21.

Essoka A.N. and Essoka, P.A. (2014). Characterization and classification of Obudu Mountain steep hillside soils. Nigerian Journal of Soil Science, 24, pp. 1-12.

Essoka, A. N. and Essoka, P.A. (2013). Classification of the Obudu Plateau (Crest) and Sub Plateau soils of Cross Rivers State, Nigeria. Proceeding of the $37^{\text {th }}$ Annual Conference of Soil Science Society of Nigeria, Makurdi, 23(1), pp. 32-40.

Food and Agriculture Organization. (1984). Land evaluation for forestry. FAO Forestry Paper 48. Rome: Food and Agriculture Organization, pp. 123.

Food and Agriculture Organization. (1988). FAOUNESCO Soil map of the world. The legend world soil resources. Report 60. Rome: Food and Agriculture Organization, pp. 72-74

Food and Agriculture Organization. (2006). FAO/UNESCO Soil map of the world, revised legend. World resources report 60. Rome: Food and Agriculture Organization, pp. 138

Government of Rivers State, Office of the Surveyor General (2014). Administrative Map of Khana Local Government Area showing settlements, boundaries, road networks and rivers, pp. 1

Heald, W. R. (1965). Calcium and magnesium. In: 
A.G. Norman, ed., Methods of Soil Analysis Part 2 Chemical and Microbiological Properties. Madison Wisconsin, USA: American Society of Agronomy, pp. 999-1010.

Ikhe, U.D., Gabhane, V.V., Sonune, B.A., and Damre, P.R. (2017). Assessment of yield and quality of grapes on different soils in Buldana District of Maharashtra. The Bioscan, 12(1), pp. 385393.

Jackson, M.L. (1973). Soil chemical analysis, $2^{\text {nd }}$ ed. New Delhi, India: Prentice Hall pub. Pvt Ltd., pp. 97

Mclean, E.O. (1965). Aluminium. In: C.A. Black, ed., Methods of Soil Analysis No.9 Part 2. Madison Wisconsin, USA: American Society of Agronomy, pp. 978-998.

Mohekar, D.S. (1997). Characterization of some orange growing soils of Nagpur district and their suitability evaluation. Thesis. Dr. Punjabrao Deshmukh Krishi Vidyapeeth, Akola.

Munsell colour. (1992). Munsell colour chart, Munsell colour Baltimore, M.D USA. Status of human induced soil degradation. Wageningen: ISRIC., pp. 21

Peter, K. D. and Umweni, A.S. (2020a). Morphological and physical properties of development form coastal plain sands and alluvium in Khana Local Government Area of Rivers State Southern Nigeria. African Journal of Suistainable Agricultural Development, 1(1), pp. 1-19.

Peter, K. D. and Umweni, A.S. (2020b). Characterization and classification of soils developed from coastal plain sands and alluvium in Khana Local Government Area of Rivers State, Southern Nigeria. Direct Research Journal of Agriculture and Food Science, 8(7), pp. 246-256.

Peter, K.D. and Ayolagha, G.A. (2012). Effect of remediation on growth parameters, grain and dry matter yield of soybean (Glycine max) in crude oil polluted soil in Ogoniland, Southeastern Nigeria. Asian Journal of Crop Science, 4(3), pp. 113-121.

Ridolfi, L., D'Odorico, P., and Laio, F. (2006). Effect of vegetation-water table feedback on the stability and resilience of plant ecosystem. Water Resource Research, 42(1),pp. 1-5

Rossiter, D.G. (1996). A theoretical frame work for land evaluation. Geoderma, 72(3-4), pp. 165-190.

Sereke, F. (2002). Land evaluation for sustainable highland agriculture in $\mathrm{N} \mathrm{W}$-Thailand (Pang Ma Pha) with special respect to soil and water resources. Diploma Thesis. Su Hgart Institute for Soil Science, University of Hohenheim.

Thurow, T.L. and Smith, J.E. (1998). Assessment of soil and water conservation methods applied to the cultivation of steep lands of southern Honduras. Texas A\&M University, Technical Bulletin No.98-2.

Udo, E.J. and Ogunwale, J.O. (1986). Laboratory manual for the analysis of soil, plant and water samples. $2^{\text {nd }}$ ed., Ibadan: Department of Agronomy, University of Ibadan, pp. 183

United States Department of Agriculture. (2014). Keys to soil taxonomy. $12^{\text {th }}$ ed., USA, Washington DC: United States Department of Agriculture, Natural Resources Conservation Service.

Walkley, A. and Black, I.A. (1934). An examination of the Degtjareff method of determining soil organic matter, and a proposed modification of the chromic acid titration method. Soil science, 37(1), pp. 29-38.

World Reference Base for Soil Resources. (2014). International soil classification System for naming soils and creating legends for soil maps, world soil reports no. 106. Rome: Food and Agriculture Organization, pp. 13-22 